\title{
General Psychiatry Schizophrenia with and without obsessive-compulsive symptoms: a comparative analysis of performance on trail making test and disability on WHODAS
}

\author{
Apala Singh, ${ }^{1}$ Ram Pratap Beniwal (D) ,2 Triptish Bhatia, ${ }^{2}$ \\ Smita Neelkanth Deshpande ${ }^{2}$
}

To cite: Singh A, Beniwal RP, Bhatia T, et al. Schizophrenia with and without obsessivecompulsive symptoms: a comparative analysis of performance on trail making test and disability on WHODAS. General Psychiatry 2020;33:e100237. doi:10.1136/ gpsych-2020-100237

AS and RPB contributed equally.

AS and RPB are joint first authors.

Received 09 April 2020

Revised 30 July 2020

Accepted 04 August 2020

\section{Check for updates}

(C) Author(s) (or their employer(s)) 2020. Re-use permitted under CC BY-NC. No commercial re-use. See rights and permissions. Published by BMJ.

${ }^{1}$ Department of Psychiatry, Govind Ballabh Pant Institute of Postgraduate Medical Education and Research (G.I.P.M.E.R.), New Delhi, Delhi, India

${ }^{2}$ Department of Psychiatry \& Drug De-addiction, Centre of Excellence in Mental Health, Atal Bihari Vajpayee Institute of Medical Sciences (ABVIMS) \& Dr Ram Manohar Lohia Hospital, New Delhi, Delhi, India

Correspondence to Dr Ram Pratap Beniwal; beniwal_9@yahoo.co.in

\section{ABSTRACT}

Background Presence of obsessive-compulsive symptoms (OCS) affects performance on tests of some cognitive functions, such as the trail making test (TMT), and may affect the level of disability in schizophrenia (SZ). Aims The aim of the present study was to compare performance on TMT and disability on WHO Disability Assessment Schedule (WHODAS) in persons with SZ with and without OCS in a cross-sectional study.

Methods Persons with SZ $(n=200)$ fulfilling DSM-V (Diagnostic and Statistical Manual) diagnostic criteria were assessed on Yale Brown Obsessive Compulsive Scale (YBOCS) and divided into two groups based on presence or absence of OCS. TMT and WHODAS V.2.0 were applied. The two groups as a whole, as well as a subsample matched on age, gender and age of onset were compared. Results Out of 200 persons with SZ, 37 (18.5\%) reported OCS. The OCS group took a significantly longer mean time to complete TMT-A ( $Z=-3.02, p=0.003)$ as well as TMT-B ( $Z=-3.551, p<0.001)$. Significant correlations were found between TMT-A and total YBOCS Scores $(r=0.351$, $\mathrm{p}=0.033$ ), as well as TMT-A and YBOCS compulsion scores $(r=0.404, p=0.013)$ but not with TMT-B Scores. The OCS group reported greater disability in all domains separately as well as on average WHODAS Scores $\left(Z=-5.969_{\text {, }}\right.$ $p<0.001)$. Significant correlations were found between YBOCS obsession scores and YBOCS total scores with average WHODAS Scores $(r=0.614, p<0.001$ and $r=0.406$, $p=0.013$, respectively). We obtained essentially similar results with the matched subsample as well as with the entire group.

Conclusion Persons with SZ and comorbid OCS had significantly poorer performance on TMTs and greater disability in comparison to persons with SZ alone. Magnitude of disability correlated with severity of OCS.

\section{INTRODUCTION}

Schizophrenia (SZ) is associated with significant generalised disability ${ }^{1}$ and marked impairment in memory, attention and executive or frontal lobe functions. Executive impairment affects planning and initiation of tasks, selection of correct actions, suppression of responses that are not appropriate to the situation and monitoring actions already undertaken. ${ }^{2}$ Impaired everyday functioning in people with SZ spans the major functional domains of independence in residence, productive activities and social functioning. ${ }^{3}$

Similarly, obsessive-compulsive disorder (OCD) is one of the most disabling medical conditions worldwide with a lifetime prevalence of $2.5 \%$ in the general population. ${ }^{4}$ A weighted prevalence rate of $3.1 \%$ was reported in a meta-analysis that included prevalence studies from India. ${ }^{5}$ The disorder results in impairment in functioning and poor quality of life with disability in several areas, particularly marital, occupational, emotional and social functioning. ${ }^{6} 7$ The primary neuropsychological deficit in $\mathrm{OCD}$ is executive dysfunction, in the form of perseverative and repetitive behaviour, difficulty in set-shifting and response inhibition. ${ }^{38}$ Contrarily, another set of studies suggested no difference on set-shifting between persons with OCD and healthy controls. ${ }^{9} 10$ Patients with OCD performed worse than healthy controls on trail making tests (TMTs) A and $\mathrm{B}$ in one study, ${ }^{11}$ while another reported no significant difference. ${ }^{12}$

Persons with comorbid SZ and OCS showed delayed non-verbal memory and cognitive shifting abilities and performed worse in the areas of visuospatial skills than those with SZ alone. ${ }^{13}$ It was hypothesised that the effect of OCS in SZ may depend on the stage of illness, with OCS conferring greater impairment in the chronic phase but possibly a protective effect early in the course of SZ. ${ }^{14}$

Persons with SZ with comorbid OCS showed more deficits in tasks measuring visuospatial 
perception and visual memory, cognitive shifting abilities, immediate verbal learning and had higher perseveration with increasing effect sizes over time. Persistent associations between the severity of comorbid OCS and impaired performance in these cognitive domains was reported. ${ }^{15}$ On tests of executive functioning and memory tasks, there was a gradual increase in cognitive dysfunction from OCD to OCD with poor insight, SZ with OCD and SZ alone. ${ }^{16}$

The level of quality of life of SZ patients with comorbidity of OCS was lower but no correlation was found between OCS and neurocognition and quality of life. ${ }^{17}$ Poorer clinical course with lower level of function especially for self-care and social competency was reported, but in only 10 patients with SZ with comorbid OCD. ${ }^{18}$ Others reported that 'schizo-obsessive' patients had significantly more impairment in social behaviour, ${ }^{19}$ as opposed to a higher level of functioning in patients of SZ with comorbid OCD. ${ }^{20}$

Thus, presence of OCS may modify cognitive functioning and degree of disability in SZ in different ways. This study was undertaken to assess and compare performance on TMT and disability on the WHO Disability Assessment Scale (WHODAS) V.2.0, in SZ subjects with/ without OCS.

\section{MATERIALS AND METHOD}

\section{Study design}

Persons with SZ with or without a caregiver, aged 18-60 years, of both gender, receiving treatment from the outpatient department of psychiatry, of a tertiary carefree public hospital between November 2016 and March 2018 were requested for participation by their psychiatrists. With no history of substance use except for nicotine use and absence of any other disorder interfering with diagnosis were included in the study. A formal written informed consent was obtained and diagnosis was established using DSM-5 criteria (figure 1).

The Yale Brown Obsessive Compulsive Scale (YBOCS) was applied on all the subjects to assess for OCS, followed by TMT A and B; the WHO Disability Assessment Schedule V.2.0 (WHODAS 2.0) was applied to assess the level of disability in all the subjects. The primary assessor (AS) was trained extensively on the scales to be used in the study by psychiatrists experienced in their use. All assessments were also reviewed in meetings with a board-certified psychiatrist who was well versed with these procedures.

\section{Assessment instruments}

\section{Yale Brown Obsessive Compulsive Scale}

YBOCS is widely used in research and clinical practice to assess the severity of OCD as well as to monitor improvement during treatment. ${ }^{21}$ It is a semistructured scale consisting of 10 core items measuring time, interference, distress, resistance and control of obsessions (items 1-5) and 5 identical items measuring compulsions (items 6-10). The items are rated from 0 (no symptoms) to 4 (severe symptoms). There are six additional

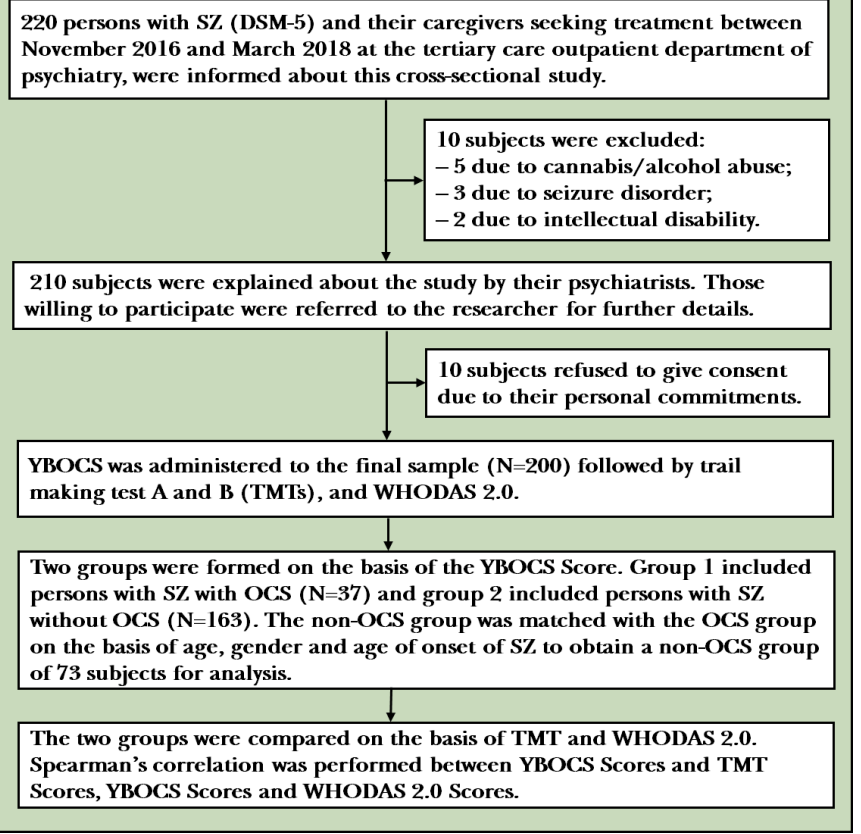

Figure 1 Flow chart of the study design. OCS, obsessivecompulsive symptoms; SZ, schizophrenia; TMT, trail making test; WHODAS 2.0, WHO Disability Assessment Schedule V.2.0; YBOCS, Yale Brown Obsessive Compulsive Scale.

investigational items (insight, avoidance, indecisiveness, pathological responsibility, pathological slowness and pathological doubting), which are not included in the total score. For the purpose of this study, we evaluated YBOCS items for the past 1 week.

\section{Trail making test}

The test is done in two parts: trail making, part A (TMT-A) involves drawing a line connecting consecutive numbers from 1 to 25. Part B (TMT-B) involves connecting alternating numbers and letters in sequence. The time to complete each 'trail' is recorded. The test administrator points out errors as they occur, and error correction influences the time to complete a trail. ${ }^{22}$ TMT-A assesses information processing, attention, visual scanning and eye-hand coordination. ${ }^{11}$ TMT-B consists of circles, some of which contain numbers and others, letters. The alternation between serial sequences of letters and numbers is said to require executive control, specifically, flexibility of thinking and greater use of working memory. ${ }^{23}$ TMT-B involves set switching tasks and it is a clearer index of executive function. ${ }^{24}$

\section{WHO Disability Assessment Schedule V.2.0}

The WHODAS contains 36 items on functioning and disability with a recall period of 30 days covering seven domains: (1) Understanding and communication (6 items); (2) Getting around (5 items); (3) Self-care (4 items); (4) Getting along with others (5 items); (5) Life activities; household (4 items); (6) Life activities: school/work (4 items), and (7) participation in society (8 items). Response options range from 1 (no difficulty) 
Table 1 Comparison between schizophrenia with and without OCS on TMT-A and TMT-B in matched subsample by Man Whitney U test

\begin{tabular}{|c|c|c|c|c|}
\hline Time (in seconds) & OCS group $(n=37)$ & Non-OCS group $(n=73)$ & $Z$ value & $P$ value \\
\hline TMT-A & $129.57(95.52)$ & $77.18(44.63)$ & -3.02 & 0.003 \\
\hline TMT-B & $350.05(197.74)$ & 214.63 (136.22) & -3.551 & $<0.001$ \\
\hline
\end{tabular}

TMT= trail making test

to 5 (extreme difficulty/cannot do). ${ }^{25}$ The DSM- 5 Disability Study Group recommended WHODAS 2.0 as the best current measure of disability for routine clinical use and recommended its inclusion in DSM-5. Assessment of disability by WHODAS is not based on diagnostic considerations. It can be used for any medical or psychiatric illness and does not depend on the aetiology of the impairment. ${ }^{2627}$ It is a standardised cross-cultural instrument with robust psychometric properties. ${ }^{26-28}$ Thirty-six-item versions of WHODAS 2.0 are available as self-administered, proxy administered and interviewer administered. ${ }^{25}$ The 36 -item version was used in the present study. Disability was assessed for the past 1 month.

The sample size $(n=200)$ was calculated for the original study (Singh et al.). ${ }^{29}$ Out of a total of 200 participants, 37 included persons with SZ with OCS and 163 was nonOCS. The OCS sample was matched with the non-OCS sample on gender, age and age of onset of SZ in a ratio of 1:2. Thus, a non-OCS sample of 73 subjects was selected. The power was calculated using means of the two samples namely OCS $(n=37)$ and non-OCS $(n=73)$. Power was calculated separately for trail A (effect size of 0.7 ) and trail B (effect size of 0.8). It was calculated as $94 \%$ and $96.5 \%$, respectively.

\section{Data analysis}

At the end of the data collection, two groups were formed, one with SZ only $(n=163)$ and the other, SZ with comorbid OCS $(n=37)$. Out of 200 subjects, $18.5 \%(n=37)$ reported OCS. First, we compared the entire non-OCS

\begin{tabular}{|c|c|c|c|}
\hline Time (seconds) & $\begin{array}{l}\text { To } \\
\text { (Total } \\
\text { obsession } \\
\text { score) }\end{array}$ & $\begin{array}{l}\text { Tc } \\
\text { (Total } \\
\text { compulsion } \\
\text { score) }\end{array}$ & $\begin{array}{l}\text { To + Tc } \\
\text { (Total } \\
\text { YBOCS } \\
\text { Score) }\end{array}$ \\
\hline \multicolumn{4}{|l|}{ TMT-A } \\
\hline $\begin{array}{l}\text { Spearman's } \\
\text { correlation, r }\end{array}$ & 0.142 & 0.404 & 0.351 \\
\hline $\mathrm{P}$ value & 0.400 & 0.013 & 0.033 \\
\hline \multicolumn{4}{|l|}{ TMT-B } \\
\hline $\begin{array}{l}\text { Spearman's } \\
\text { correlation, r }\end{array}$ & 0.253 & 0.148 & -0.006 \\
\hline $\mathrm{P}$ value & 0.13 & 0.384 & 0.971 \\
\hline
\end{tabular}

Bold value of 0.404 and 0.351 denotes statistically significant at $\mathrm{p}<0.05$.

YBOCS, Yale Brown Obsessive Compulsive Scale. group $(n=163)$ with the OCS $(n=37)$ group. In the second step, we compared the non-OCS subsample $(n=73$ of the original 163 subjects after matching for age, sex and age of onset of SZ) with the OCS group $(n=37) .^{29}$

Using SPSS V.21 (IBM Corp, released 2012), data were checked for normality and analysed. ${ }^{30}$ Continuous variables were calculated as mean (SD) and categorical variables as absolute numbers and percentages. Normally distributed continuous variables were compared using the unpaired t-test. Mann-Whitney $U$ test was used for non-normally distributed variables. Categorical variables were analysed using either the $\chi^{2}$ test or Fisher's exact test. Spearman's correlation was calculated among various parameters. For all statistical tests, a value of $\mathrm{p}<0.05$ was taken to indicate a statistically significant difference.

\section{RESULTS}

During the course of the study, 220 persons with SZ, who visited for treatment, were requested for participation after due referral by the psychiatrist. The majority were accompanied by a caregiver, usually a relative residing with the participant, who also contributed actively to assessment interviews. Out of these, 20 were excluded due to refusal to give consent and presence of comorbidities. A total of 200 persons with SZ was available for final analysis. After YBOCS administration, three groups were formed; group 1: SZ without OCS/OCD $(n=163)$, group 2: SZ with OCS ( $n=14$, YBOCS Score $<16)$ and group 3: SZ with OCD $(n=23$, YBOCS Score $\geq 16)$. As groups 2 and 3 were very small as compared with group 1 , we combined these two $(n=37)$ as defined by Devi. ${ }^{31}$ We matched group 1 with group 2 samples for age, gender and age of onset of SZ. There were 73 matched cases that were included in the non-OCS group.

Results of demographic details, age of onset and total duration of illness (TDI) are presented for the entire sample $(n=200)$ between the OCS group $(n=37)$ and the non-OCS group $(n=163)$. Results showing the comparison of performance on TMT and disability are presented for the OCS group $(n=37)$ and the matched subsample $(n=73)$. We obtained essentially similar results with the matched subsample as with the entire group.

There were 112 men $(56.0 \%)$ and 88 women $(44.0 \%)$ with mean age of 35.05 (10.19) years . Majority were married $(\mathrm{n}=106,53.0 \%)$ and residing in an urban area $(n=185,92.5 \%)$. Most $(n=121,60.5 \%)$ were unemployed. Mean age of onset of SZ was 25.57 (8.782) years. Mean total TDI was $9.22(7.303)$ years. 
Table 3 Comparison between schizophrenia with and without OCS on WHODAS in a matched subsample

\begin{tabular}{|c|c|c|c|c|}
\hline WHODAS Scores & $\begin{array}{l}\text { OCS group } \\
(n=37)\end{array}$ & $\begin{array}{l}\text { Non-OCS group } \\
(n=73)\end{array}$ & $Z$ value & $\mathbf{P}$ value \\
\hline $\begin{array}{l}\text { D1-Average score of } \\
\text { Domain } 1 \text { of WHODAS }\end{array}$ & $3.02(0.85)$ & $2.21(0.73)$ & -4.663 & $<0.001$ \\
\hline $\begin{array}{l}\text { D2-Average score of } \\
\text { Domain } 2 \text { of WHODAS }\end{array}$ & $1.45(0.83)$ & $1.12(0.22)$ & -4.638 & $<0.001$ \\
\hline $\begin{array}{l}\text { D3-Average score of } \\
\text { Domain } 3 \text { of WHODAS }\end{array}$ & $1.43(0.45)$ & $1.15(0.23)$ & -3.98 & $<0.001$ \\
\hline $\begin{array}{l}\text { D4-Average score of } \\
\text { Domain } 4 \text { of WHODAS }\end{array}$ & $3.25(0.77)$ & $2.11(0.85)$ & -5.846 & $<0.001$ \\
\hline $\begin{array}{l}\text { D5(1)-Average score of } \\
\text { Domain } 5 \text { (1) of WHODAS }\end{array}$ & $3.14(1.13)$ & $2.1(0.88)$ & -4.485 & $<0.001$ \\
\hline $\begin{array}{l}\text { D5(2)-Average score of } \\
\text { Domain } 5 \text { (2) of WHODAS }\end{array}$ & $3.73(0.79)$ & $2.1(0.86)$ & -4.219 & $<0.001$ \\
\hline D total Average score & $2.69(0.59)$ & $1.85(0.51)$ & -5.969 & $<0.001$ \\
\hline
\end{tabular}

D1=Cognition, D2=Mobility, D3=Self care, D4=Getting along with people, D5 (1)=Household activities, D5 (2)=Work or school activities, D6=Participation.

The mean age of all participants $(n=200)$ was compared using Student's t-test. The mean age of the OCS group was 36.22 (10.26) years and that of the non-OCS group was $34.79(10.18)$ years. There were $90(55.2 \%)$ and 22 $(59.5 \%)$ men in the non-OCS group and the OCS group, respectively. The non-OCS group had 73 (44.8\%) women and the OCS group had $15(40.5 \%)$ women. There was no significant difference in mean age, gender, marital status and residence between the two groups using the $\chi^{2}$ test.

The Mann-Whitney $U$ test was applied to compare TDI and age of onset. The mean TDI of the non-OCS group was 8.71 (6.83) years and that of the OCS group was 11.46 (8.87) years. The mean age of onset of SZ in the non-OCS group was 25.88 (8.78) years, while that in the OCS group was 24.2 (8.78) years. No significant differences were present between the two groups on TDI and age of onset.

Mean time taken to complete TMT-A was 92.82 (68.325) seconds and TMT-B was 251.5 (164.283) seconds in the sample as a whole $(n=200)$. Mann-Whitney $U$ test was used to compare the time taken to complete TMT-A and TMT-B between the OCS group $(n=37)$ and the matched non-OCS subsample $(n=73)$. The OCS group took significantly more time for TMT-A $(\mathrm{Z}=-3.02, \mathrm{p}=0.003)$ and TMT-B $(\mathrm{Z}=-3.551, \mathrm{p}=<0.001)$ (table 1$)$. This suggests that persons with SZ with comorbid OCS performed more poorly on TMT than persons with SZ alone.

Among persons with SZ and OCS, correlation was calculated separately for compulsion and obsession scores on YBOCS and the time taken to complete TMT-A and TMT-B. There was significant positive correlation between YBOCS compulsion score (Tc) and TMT-A $(\mathrm{r}=0.404, \mathrm{p}=0.013)$ as well as significant positive correlation between total YBOCS scores (To + Tc) and TMT-A $(\mathrm{r}=0.351, \mathrm{p}=0.033)$. This suggests that the more severity of OCS, the more impairment on TMT-A. However, there was no significant correlation between YBOCS scores and TMT-B (table 2).

For each participant, total disability score was calculated by summing individual domain scores. Average scores for each domain (D1 average, D2 average...) were calculated by dividing the total domain scores (D1 total, D2 total...) by number of items answered in each domain. Average score for WHODAS total score was calculated by dividing the total score (D total) by number of items answered in the entire scale. The mean WHODAS total score was 69.46 (20.33) and the mean WHODAS total average scores was $2.07(0.597)$ in the entire sample $(n=200)$. In the matched subsample, total average WHODAS Scores were compared between the two groups using Mann Whitney U test. The OCS group scored significantly higher on total average WHODAS Score $(Z=-5.969, p \leq 0.001)$ as well as on each domain separately (table 3 ).

We considered average scores (domain as well as total) for calculations, taking into account missing items in some of the domains (table 4). Correlations were performed among YBOCS obsession scores (To), YBOCS compulsion scores (Tc) and total YBOCS scores (To + Tc) with average WHODAS scores (domain and total). There was significant correlation between YBOCS total scores (To $+\mathrm{Tc}$ ) and average of domains D1, D4, D5 (1), D5 (2). There was also significant positive correlation between YBOCS total scores and WHODAS total average score $(\mathrm{r}=0.406$, $\mathrm{p}=0.013$ ). YBOCS obsession scores (To) significantly positively correlated with each of the average WHODAS domain scores and with WHODAS total average score $(\mathrm{r}=0.614, \mathrm{p} \leq 0.001)$. However, significant correlation was not found between YBOCS compulsion scores and any of the WHODAS scores. 
Table 4 Correlations between YBOCS Scores and WHODAS Scores $(n=37)$

\begin{tabular}{|c|c|c|c|}
\hline WHODAS scores & $\begin{array}{l}\text { To } \\
\text { (Total obsession score) }\end{array}$ & $\begin{array}{l}\text { Tc } \\
\text { (Total compulsion score) }\end{array}$ & $\begin{array}{l}\text { To + Tc } \\
\text { (Total YBOCS score) }\end{array}$ \\
\hline \multicolumn{4}{|l|}{$\begin{array}{l}\text { D1-Average score of } \\
\text { Domain } 1 \text { of WHODAS }\end{array}$} \\
\hline Spearman's correlation, $r$ & 0.469 & 0.215 & 0.358 \\
\hline$P$ value & 0.003 & 0.202 & 0.029 \\
\hline \multicolumn{4}{|l|}{$\begin{array}{l}\text { D2-Average score of } \\
\text { Domain } 2 \text { of WHODAS }\end{array}$} \\
\hline Spearman's correlation, $r$ & 0.333 & 0.109 & 0.229 \\
\hline$P$ value & 0.044 & 0.522 & 0.173 \\
\hline \multicolumn{4}{|l|}{$\begin{array}{l}\text { D3-Average score of } \\
\text { Domain } 3 \text { of WHODAS }\end{array}$} \\
\hline Spearman's correlation, $r$ & 0.370 & 0.085 & 0.230 \\
\hline$P$ value & 0.024 & 0.616 & 0.171 \\
\hline \multicolumn{4}{|l|}{$\begin{array}{l}\text { D4-Average score of } \\
\text { Domain } 4 \text { of WHODAS }\end{array}$} \\
\hline Spearman's correlation, $r$ & 0.484 & 0.313 & 0.437 \\
\hline$P$ value & 0.002 & 0.060 & 0.007 \\
\hline \multicolumn{4}{|l|}{$\begin{array}{l}\text { D5(1)-Average score of } \\
\text { Domain } 5 \text { (1) of WHODAS }\end{array}$} \\
\hline Spearman's correlation, $r$ & 0.544 & 0.177 & 0.363 \\
\hline$P$ value & 0.001 & 0.296 & 0.027 \\
\hline \multicolumn{4}{|l|}{$\begin{array}{l}\text { D5(2)-Average score of } \\
\text { Domain } 5 \text { (2) of WHODAS }\end{array}$} \\
\hline Spearman's correlation, $r$ & 0.838 & 0.406 & 0.652 \\
\hline$P$ value & 0.001 & 0.190 & 0.021 \\
\hline \multicolumn{4}{|l|}{$\begin{array}{l}\text { D6-Average score of } \\
\text { Domain } 6 \text { of WHODAS }\end{array}$} \\
\hline Spearman's correlation, $r$ & 0.549 & 0.068 & 0.293 \\
\hline$P$ value & $<0.001$ & 0.691 & 0.078 \\
\hline \multicolumn{4}{|l|}{ D-total Average score } \\
\hline Spearman's correlation, $r$ & 0.614 & 0.189 & 0.406 \\
\hline$P$ value & $<0.001$ & 0.263 & 0.013 \\
\hline
\end{tabular}

Bold values are statistically significant at $p<0.05$ level.

${ }^{*}$ D1 = Cognition, D2 = Mobility, D3 = Self-care, D4 = Getting along with people, D5(1) = Householdactivities, D5(2) = Work or school activities, D6 = Participation

\section{DISCUSSION}

\section{Main findings}

The mean age of onset of subjects in our study was 25.57 years, comparable to earlier studies. ${ }^{19} 31-33$ The mean total duration of $\mathrm{SZ}$ was 9.22 years, similar to a previous study. ${ }^{34}$ There were no significant differences in age, gender, marital status or residence between the OCS and nonOCS groups, again comparable to past studies. ${ }^{173435}$

OCS group took significantly longer time than the nonOCS group to complete both TMT-A and TMT-B. The OCS group had significantly poorer processing speed and poorer executive function, comparable to some earlier studies, ${ }^{13} 1836$ but not others. ${ }^{1737}$ Lee et al. ${ }^{35}$ in a small sample $(n=27)$, reported better executive function in their OCD group. ${ }^{35}$ Our study did not find significant correlation between YBOCS scores and TMT-B (which measures executive function); though significant positive correlation between TMT-A with YBOCS compulsion scores and total scores was found, previous studies have reported contradictory findings. ${ }^{15}$

Our Schizophrenia OCS (SZ-OCS) group showed greater disability in all domains of WHODAS 2.0. Total YBOCS obsession scores significantly correlated with total average WHODAS disability score as well as with each domain individually. Total YBOCS scores also correlated significantly with all WHODAS domains and average scores except domains of getting around (mobility), selfcare and participation.

Disability in social and occupational areas has been earlier reported in both $\mathrm{OCD}$ and $\mathrm{SZ}^{38} 39$ with greater disability in those with both conditions, along with significantly poorer quality of life. ${ }^{17}$ However, some studies 
demonstrated better functioning, perhaps because of fewer negative symptoms. ${ }^{20}$ Devi ${ }^{31}$ suggested that better environmental quality of life (QOL) may be due to the greater number of subjects with urban residence in their OCS/OCD group. ${ }^{31}$

Hence, our study results emphasize the importance of comorbid OC symptoms in schizophrenia. ${ }^{40}$ Pharmacotherapy should be combined with cognitive-behavioural therapy to treat OCS in SZ once the patient is cooperative to participate in the therapy. ${ }^{41}$

\section{Limitations and strengths}

However, we had a larger male, urban and unemployed sample with no restriction as to the stage or duration of illness. This might have led to a confounding effect due to the chronicity and severity of illness on our assessments. There was no restriction as to the treatment status, type of medications used, their duration, dose etc., which could have influenced our results. Treatment emergent OCS were not excluded in our study. The strengths of our study are a larger sample size and analysing a matched subsample.

\section{Implications}

Patients with SZ should be screened for the presence of OCS. Residents should be trained to identify and differentiate OCS from the psychotic symptoms in SZ. Our study showed poorer performance on TMT and greater disability on WHODAS in subjects of SZ with comorbid OCS. Timely recognition and treatment of these symptoms may lead to better cognitive outcomes and less dysfunction in these patients. It is recommended that future studies take into account the influence of untreated $\mathrm{SZ}$ as well as treatment with antipsychotics on emergence and severity of OCS.

\section{Twitter Ram Pratap Beniwal @RamPratapBeniw4}

Acknowledgements The authors thank the participants and members of their department for their help and support towards completing this study and paper. The authors also thank Dr Rajesh Nagpal for his expert inputs on reliability.

Contributors AS: Data acquisition, data analysis, review of literature and preparation of the manuscript. RPB: Design, data acquisition and analysis, manuscript writing and editing and critical review. TB: Concept, manuscript review and editing. SND: Concept, design, manuscript editing and review. All the coauthors checked the manuscript for language, analysis and interpretation of data.

Funding The authors have not declared a specific grant for this research from any funding agency in the public, commercial or not-for-profit sectors.

Competing interests None declared.

Patient consent for publication Not required.

Ethics approval The study received ethical approval and permission from the Institutional Ethics Committee and Institutional Review Board of the same institute: Institutional Ethics committee-ABVIMS-Dr RMLH, New Delhi, India (Number/ID: TP(MD/MS)(21/2016)/IEC/PGIMER/RMLH/7795/16).

Provenance and peer review Not commissioned; externally peer reviewed.

Data availability statement Data would be available upon reasonable request to the corresponding author for the paper through email.

Open access This is an open access article distributed in accordance with the Creative Commons Attribution Non Commercial (CC BY-NC 4.0) license, which permits others to distribute, remix, adapt, build upon this work non-commercially, and license their derivative works on different terms, provided the original work is properly cited, appropriate credit is given, any changes made indicated, and the use is non-commercial. See: http://creativecommons.org/licenses/by-nc/4.0/.

\section{ORCID iD}

Ram Pratap Beniwal http://orcid.org/0000-0001-6762-3702

\section{REFERENCES}

1 Schaefer J, Giangrande E, Weinberger DR, et al. The global cognitive impairment in schizophrenia: consistent over decades and around the world. Schizophr Res 2013;150:42-50.

2 Liddle PF. Cognitive impairment in schizophrenia:its impact on social functioning. Acta Psychiatr Scand 2000;101:11-16.

3 Olley A, Malhi G, Sachdev P. Memory and executive functioning in obsessive-compulsive disorder: a selective review. J Affect Disord 2007;104:15-23.

4 Karno M, Golding JM, Sorenson SB, et al. The epidemiology of obsessive-compulsive disorder in five us communities. Arch Gen Psychiatry 1988;45:1094-9.

5 Reddy VM, Chandrashekar CR. Prevalence of mental and behavioural disorders in India : a meta-analysis. Indian J Psychiatry 1998;40:149-57.

6 Moritz S, Rufer M, Fricke S, et al. Quality of life in obsessivecompulsive disorder before and after treatment. Compr Psychiatry 2005;46:453-9.

7 Bystritsky A, Liberman RP, Hwang S, et al. Social functioning and quality of life comparisons between obsessive-compulsive and schizophrenic disorders. Depress Anxiety 2001;14:214-8.

8 Boone KB, Ananth J, Philpott L, et al. Neuropsychological characteristics of Nondepressed adults with obsessive-compulsive disorder. Cognitive and Behavioral Neurology 1991;4:96.

9 Abbruzzese M, Bellodi L, Ferri S, et al. Frontal lobe dysfunction in schizophrenia and obsessive-compulsive disorder: a neuropsychological study. Brain Cogn 1995;27:202-12.

10 Christensen KJ, Kim SW, Dysken MW, et al. Neuropsychological performance in obsessive-compulsive disorder. Biol Psychiatry 1992;31:4-18.

11 Kamaradova D, Hajda M, Prasko J, et al. Cognitive deficits in patients with obsessive-compulsive disorder electroencephalography correlates. Neuropsychiatr Dis Treat 2016;12:1119-25.

12 de Geus F, Denys DAJP, SITSKOORN MM, et al. Attention and cognition in patients with obsessive?compulsive disorder. Psychiatry Clin Neurosci 2007;61:45-53.

13 Berman I, Merson A, Viegner B, et al. Obsessions and compulsions as a distinct cluster of symptoms in schizophrenia: a neuropsychological study. J Nerv Ment Dis 1998;186:150-6.

14 Borkowska A, Pilaczyñska E, Rybakowski JK. The frontal lobe neuropsychological tests in patients with schizophrenia and/or obsessive-compulsive disorder. J Neuropsychiatry Clin Neurosci 2003;15:359-62.

15 Schirmbeck F, Rausch F, Englisch S, et al. Stable cognitive deficits in schizophrenia patients with comorbid obsessive-compulsive symptoms: a 12-month longitudinal study. Schizophr Bull 2013;39:1261-71.

16 Tumkaya S, Karadag F, Oguzhanoglu NK, et al. Schizophrenia with obsessive-compulsive disorder and obsessive-compulsive disorder with poor insight: a neuropsychological comparison. Psychiatry Res 2009;165:38-46.

17 Tiryaki A, Özkorumak E. Do the obsessive-compulsive symptoms have an effect in schizophrenia? Compr Psychiatry 2010;51:357-62.

18 Hwang MY, Morgan JE, Losconzcy MF. Clinical and neuropsychological profiles of obsessive-compulsive schizophrenia: a pilot study. J Neuropsychiatry Clin Neurosci 2000;12:91-4.

19 Poyurovsky M, Hramenkov S, Isakov V, et al. Obsessive-Compulsive disorder in hospitalized patients with chronic schizophrenia. Psychiatry Res 2001;102:49-57.

20 Tibbo P, Warneke L. Obsessive-Compulsive disorder in schizophrenia: epidemiologic and biologic overlap. J Psychiatry Neurosci 1999;24:15-24.

21 Goodman WK, Price LH, Rasmussen SA, et al. The Yale-Brown obsessive compulsive scale. I. development, use, and reliability. Arch Gen Psychiatry 1989;46:1006-11.

22 Lezak MD. Neuropsychological assessment. 3rd ed. New York, NY, US: Oxford University Press, 1995: xviii, 1026. 
23 Bhatia T, Garg K, Pogue-Geile M, et al. Executive functions and cognitive deficits in schizophrenia: comparisons between probands, parents and controls in India. J Postgrad Med 2009;55:3-7.

24 Arbuthnott K, Frank J, Test TM. Trail making test, part B as a measure of executive control: validation using a set-switching paradigm. J Clin Exp Neuropsychol 2000;22:518-28.

25 Gold LH. Dsm-5 and the assessment of functioning: the world Health organization disability assessment schedule 2.0 (WHODAS 2.0). J Am Acad Psychiatry Law 2014;42:173-81.

26 Üstün TB, Kostanjsek N, Chatterji S, et al. Measuring health and disability: manual for WHO Disability Assessment Schedule [WHODAS 2.0]. [Internet]., 2010. Available: https://www.cabdirect. org/cabdirect/abstract/20133042092 [Accessed 21 Sep 2018].

27 Federici S, Meloni F, Lo Presti A. International literature review on WHODAS II [World Health Organization Disability Assessment Schedule II]. Life Span and Disability 2009;12:83-110.

28 Federici S. WHODAS II: disability self-evaluation in the ICF conceptual frame n.d.:23.

29 Singh A, Beniwal RP, Bhatia T, et al. Prevalence and clinical correlations of obsessive-compulsive symptoms in schizophrenia. Asian J Psychiatr 2019;39:48-52.

30 IBM Corp. Released [2012]. IBM SPSS Statistics for Windows, Version 21.0. Armonk, NY IBM Corp. - References - Scientific Research Publishing [Internet]. Available: http://www.scirp.org/ [S[vtj3fa45qm1ean45vvffcz55]] /reference/ReferencesPapers.aspx? ReferencelD=1311807 [Accessed 23 Sep 2018].

31 Devi S, Rao NP, Badamath S, et al. Prevalence and clinical correlates of obsessive-compulsive disorder in schizophrenia. Compr Psychiatry 2015;56:141-8.
32 Singh A, Beniwal RP, Kukshal P, et al. A preliminary study of association of genetic variants with early response to olanzapine in schizophrenia. Indian J Psychiatry 2018;60:10-16.

33 Sharma A, Kumar A, Singh S, et al. Altered resting state functional connectivity in early course schizophrenia. Psychiatry Res 2018;271:17-23.

34 Rajkumar RP, Reddy YCJ, Kandavel T. Clinical profile of "schizoobsessive" disorder: a comparative study. Compr Psychiatry 2008;49:262-8.

35 Lee M-J, Shin Y-B, Sunwoo Y-K, et al. Comparative analysis of cognitive function in schizophrenia with and without obsessive compulsive disorder. Psychiatry Investig 2009;6:286-93.

36 Lysaker PH, Marks KA, Picone JB, et al. Obsessive and compulsive symptoms in schizophrenia: clinical and neurocognitive correlates. $J$ Nerv Ment Dis 2000;188:78-83.

37 Ongür D, Goff DC. Obsessive-Compulsive symptoms in schizophrenia: associated clinical features, cognitive function and medication status. Schizophr Res 2005;75:349-62.

38 Bobes J, González MP, Bascarán MT, et al. Quality of life and disability in patients with obsessive-compulsive disorder. European Psychiatry 2001;16:239-45.

39 Guilera G, Gómez-Benito J, Pino O, et al. Utility of the world Health organization disability assessment schedule II in schizophrenia. Schizophr Res 2012;138:240-7.

40 Singh A, Beniwal RP, Bhatia T, et al. The authors respond. Asian J Psychiatr 2019;40:45.

41 Green Al, Canuso CM, Brenner MJ, et al. Detection and management of comorbidity in patients with schizophrenia. Psychiatr Clin North Am 2003;26:115-39.

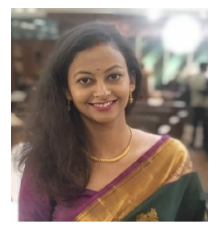

Dr. Apala Singh has done her MBBS from Maulana Azad Medical College, New Delhi, in 2014, and MD in Psychiatry from Atal Bihari Vajpayee Institute of Medical Sciences and Dr. Ram Manohar Lohia Hospital, New Delhi, India, in 2019. She has been a senior resident at the Department of Psychiatry, Govind Ballabh Pant Institute of Post Graduate Medical Education and Research, New Delhi, India, since 2020. She has attended various national and international conferences. Her main research interests include schizophrenia, $O C D$ and psychopharmacology. 